\title{
ARTICLE Predicted secreted protein analysis reveals synaptogenic function of Clstn3 during WAT browning and BAT activation in mice
}

Shu-qin Chen ${ }^{1}$, Qiang Niu ${ }^{2}$, Li-ping Ju${ }^{1}$, Miriayi Alimujiang ${ }^{1}$, Han Yan ${ }^{1}$, Ning-ning Bai ${ }^{1}$, Jun $\mathrm{Xu}^{3}$, Qi-chen Fang ${ }^{1}$, Jun-feng Han ${ }^{1}$, Ying Yang ${ }^{1}$ and Wei-ping Jia $^{1}$

Promoting white adipose tissue (WAT) browning and enhancing brown adipose tissue (BAT) activity are attractive therapeutic strategies for obesity and its metabolic complications. Targeting sympathetic innervation in WAT and BAT represents a promising therapeutic concept. However, there are few reports on extracellular microenvironment remodeling, especially changes in nerve terminal connections. Identifying the key molecules mediating the neuro-adipose synaptic junctions is a key point. In this study, we used bioinformatics methods to identify the differentially expressed predicted secreted genes (DEPSGs) during WAT browning and BAT activation. These DEPSGs largely reflect changes of cytokines, extracellular matrix remodeling, vascularization, and adipocyteneuronal cross-talk. We then performed functional enrichment and cellular distribution specificity analyses. The upregulated and downregulated DEPDGs during WAT browning displayed a distinctive biological pattern and cellular distribution. We listed a cluster of adipocyte-enriched DEPSGs, which might participate in the cross-talk between mature adipocytes and other cells; then identified a synaptogenic adhesion molecule, Clstn3, as the top gene expressed enriched in both mature white and brown adipocytes. Using Q-PCR and immunohistochemistry, we found significantly increased Clstn3 expression level during WAT browning and BAT activation in mice subjected to cold exposure $\left(4^{\circ} \mathrm{C}\right)$. We further demonstrated that treatment with isoproterenol significantly increased Clstn3 and UCP1 expression in differentiated white and beige adipocytes in vitro. In conclusion, our study demonstrates that the secretion pattern was somewhat different between WAT browning and BAT activation. We reveal that Clstn3 may be a key gene mediating the neuro-adipose junction formation or remodeling in WAT browning and BAT activation process.

Keywords: WAT browning; BAT activation; Calsyntenin-3; neuro-adipose synapse; isoproterenol; obesity

Acta Pharmacologica Sinica (2019) 40:999-1009; https://doi.org/10.1038/s41401-019-0211-2

\section{INTRODUCTION}

Obesity is a major risk factor for multiple disorders, including type 2 diabetes, cardiovascular diseases, dyslipidemias, and cancer [1]. A better understanding of adipose tissue biology is crucial to identify novel therapeutic targets for the management of obesity and other metabolic complications. Adipose tissue plays central roles in energy homeostasis [2]. There are two particular types of adipose tissue: white adipose tissue (WAT), which is characterized for storing excess energy, and brown adipose tissue (BAT), which is specialized to consume energy as heat. The murine BAT depot is mainly located in the interscapular region, and the human BAT depot is concentrated in the supraclavicular and mediastinal regions [3-5]. Recent studies have identified beige or brite (brown in white) cells that exist within WAT in mice and rats as an intermediate type of adipocytes [6]. These cells acquire BAT features in response to cold exposure, including highly expressed UCP1 (uncoupling protein 1) and multilocular lipid droplets [7]. In BAT, cold exposure increases the
UCP1 expression and mitochondrial biogenesis, resulting in an increase of thermogenic capacity [8]. Adipose tissue represents a complex tissue in terms of its resident cellular composition: mature adipocytes and various other cells that comprise the stromalvascular fractions (SVFs). Mature adipocytes are specialized metabolic cells and are surrounded by various components of the extracellular matrix wherein nerve terminals coexist with nonadipose cells [9]. The microenvironment remodeling during WAT browning and BAT activation influences the structure and function of many cell types to perform vital metabolic and endocrine functions of the adipose tissue [10]. The thermogenic function of mature adipocytes (beige and brown adipocytes) has been recognized by many researchers. However, the extracellular microenvironment remodeling, particularly changes in nerve terminal connections, has received less attention.

It is noteworthy that increased sympathetic innervation in inguinal WAT and BAT after a cold challenge has been reported

\footnotetext{
${ }^{1}$ Shanghai Key Laboratory of Diabetes, Shanghai Institute for Diabetes, Shanghai Clinical Medical Centre of Diabetes, Shanghai Key Clinical Centre of Metabolic Diseases, Department of Endocrinology and Metabolism, Shanghai Jiao Tong University Affiliated Sixth People's Hospital, Shanghai 200233, China; ${ }^{2}$ National Engineering Research Center of Die and Mold CAD, Shanghai Jiao Tong University, Shanghai 200030, China and ${ }^{3}$ Department of Geriatrics, Shanghai Jiao Tong University Affiliated Sixth People's Hospital, Shanghai 200233, China

Correspondence: Ying Yang (yangyingsh@sjtu.edu.cn) or Jun-feng Han (tjhjf@163.com)

These authors contributed equally: Shu-qin Chen, Qiang Niu
}

Received: 26 August 2018 Accepted: 5 January 2019

Published online: 22 February 2019 
[11-13]. A breakthrough study recently reported that sympathetic nerve fibers can establish neuro-adipose junctions by directly forming synapse-like structures in mouse WAT, and neuro-adipose junctions are necessary and sufficient for leptin-stimulated lipolytic responses of WAT [14]. A subsequent study also indicated a dense network of sympathetic arborization in inguinal WAT using the volume fluorescence-imaging technique and confirmed that the dense sympathetic arborizations are essential for coldinduced WAT browning [11]. To date, the key molecules that mediate the neuro-adipose synaptic junctions have not been identified.

In our study, we obtained a predicted secreted protein $(n=$ 3171) list from the Tissue-Based Map Of Human Proteome [15]. A whole-proteome scan was used to predict the complete set of human secreted proteins using three methods for signal-peptide prediction: SignalP4.0 [16], Phobius [17], and SPOCTOPUS [18]. The signal peptide is a short peptide present at the N-terminal of the majority of proteins that are targeted to the endoplasmic reticulum and eventually destined to the secretory pathway [19, 20]. These proteins include those that are secreted from the cell (extracellular or periplasmic), inserted into most cellular membranes, or reside inside certain organelles (the endoplasmic reticulum, Golgi or endosomes). Thus, we suggest secretory protein changes can largely reflect the changes of cytokines, the extracellular matrix and the connections of cells, which can represent the extracellular microenvironment remodeling and may help us identify key molecules that mediate the neuroadipose junction.

Here, we identified DEPSGs during WAT browning and BAT activation using GEO DataSets and predicted secreted protein list. We subsequently compared the DEPSGs between WAT browning and BAT activation and further performed functional enrichment analysis to identify the key GO terms and pathways. In addition, we determined the cellular distribution specificity of the upregulated and downregulated secreted genes that had a neat segregated pattern. Based on these analyses and validated experiments, we identified that Clstn3 may be a key molecule in neuro-adipose synapse formation or remodeling during WAT browning and BAT activation.

The calsyntenin family has three members (Clstn1, Clstn2, and Clstn3). Clstn3 is the only one identified as a synaptogenic cell adhesion molecule (CAM) and has been confirmed to promote synapses [21, 22]. It is a transmembrane protein localized in part to the postsynaptic membrane, with extracellular domains that contain two cadherin repeats and sex hormone-binding globulin [21-23]. Previous research has mainly focused on its function in the brain, while the function of Clstn3 in peripheral tissue and organs has not been studied. Here, our work first explored its function in adipose tissue. These findings may advance our knowledge of the physiological process of WAT browning and BAT activation and may provide therapeutic enlightenment for the treatment of obesity.

\section{MATERIALS AND METHODS}

\section{Materials}

Dulbecco's modified Eagle's medium (DMEM), fetal bovine serum, penicillin/streptomycin solution and trypsin were obtained from Gibco (Grand Island, NY, USA). Type II collagenase, isobutylmethylxanthine, dexamethasone, rosiglitazone, T3, and insulin were obtained from Sigma (St Louis, MO, USA). Antibodies against UCP1 and Clstn3 were obtained from Abcam (Cambridge, MA, USA).

Data extraction and processing

The National Center for Biotechnology Information Gene Expression Omnibus (GEO, https://www.ncbi.nlm.nih.gov/geo/) is a public dataset that stores curated gene expression resources, such as array- and sequence-based data, and is freely available to users [24]. The gene expression profiles of GSE86338 by highthroughput sequencing were downloaded from GEO. The differentially expressed genes (DEGs) of GSE86338 were identified by EBSeq algorithms. Genes were considered significantly differentially expressed under the following criteria: (1) fold change $(F C)>1.2$ and (2) FDR $<0.05$.

The gene expression profile of GSE44059 by microarray was obtained from the GEO. This dataset contains gene expression data of the adipocyte and SVFs of the interscapular brown, inguinal subcutaneous and visceral epididymal adipose tissue depots of young adult male C57BL/6 [25]. GEO2R (https://www. ncbi.nlm.nih.gov/geo/geo2r/) was applied to screen DEGs between mature adipocyte and SVF samples in GSE44059 [26]. $P$-values $<0.05$ were set as the cut-off criterion.

A predicted secreted protein list was obtained from TissueBased Map of Human Proteome [15]. It is an integrative genomic resource that describes proteomes and transcriptomes across diverse tissues and organs of the human body, providing a spatial atlas of the expression for thousands of genes down to the singlecell level $[15,27]$. To build their resource, Uhlén et al. used samples from 44 human tissues (representing all major tissues and organs), each of which was probed with $\sim 24,000$ different antibodies, complemented with RNA-sequencing data for 32 of the tissues. In Uhlén's study, a whole-proteome scan was used to predict the complete set of human secreted proteins ("secretome") using three methods for signal-peptide prediction. The data from this study are freely available at http://www.proteinatlas.org, providing the possibility to aid future studies $[15,28]$.

Functional enrichment and pathway enrichment analysis GO enrichment analysis is a common useful method for the identification of overrepresented (or underrepresented) biological attributes for high-throughput genome or transcriptome data [29, 30]. Pathway enrichment analysis is a systematic analysis of gene function, and it links genomic information with functional information [31]. GO and pathway enrichment analysis was performed using the SBC analysis system (http://enrich.shbio. com/index/index.asp). The Shanghai Biotechnology Corporation (SBC) Analysis System is an online system for the analysis of microarrays and integrates various datasets that contain gene ontology, KEGG, and the BioCarta database.

Quantitative real-time polymerase chain reaction (qRT-PCR) analysis

Total RNAs were extracted using Trizol reagent (Invitrogen, Carlsbad, (A, USA), and CDNA was generated using an iScript cDNA Synthesis Kit (Bio-Rad, Hercules, CA, USA). qRT-PCR analysis was performed with a Roche LightCycler 480 system using SYBR Premix Ex Taq (Takara Bio, Shiga, Japan). The primers used to detect the expressions of target genes are listed as follows: mouse Clstn3, sense 5'-CGATACTCCAGCAACGAATTCA-3', and antisense 5'-CCAC CCGGTTCATGCTATG-3'; mouse 36B4, sense 5'-AAGCGCGTCCTGGC ATTGTCT-3', and antisense 5'-CCGCAGGGGCAGCAGTGGT-3'.

Cell culture and differentiation

3T3-L1 cell lines were purchased from the ATCC, routinely cultured in DMEM supplemented with $10 \%$ FBS and penicillin/streptomycin solution and maintained in a humidified incubator at $37^{\circ} \mathrm{C}$ and $5 \%$ $\mathrm{CO}_{2}$. For induction to white adipocytes, 3T3-L1 cells were incubated with medium that contained $5 \mu \mathrm{g} / \mathrm{mL}$ insulin, $0.5 \mathrm{mM}$ isobutylmethylxanthine, and $1 \mu \mathrm{M}$ dexamethasone for two days and were subsequently cultured with $5 \mu \mathrm{g} / \mathrm{mL}$ insulin for the following 6 days.

SVFs were isolated from the inguinal WAT of C57/BL6J mice. The inguinal WAT was minced and then digested with type II collagenase for $1 \mathrm{~h}$ at $37^{\circ} \mathrm{C}$ with gentle shaking. Digested adipose tissue was filtered through a $40 \mu \mathrm{M}$ cell strainer. After centrifugation at $500 \times g$ for $10 \mathrm{~min}$ at $4{ }^{\circ} \mathrm{C}$, the SVF cells were resuspended 
a

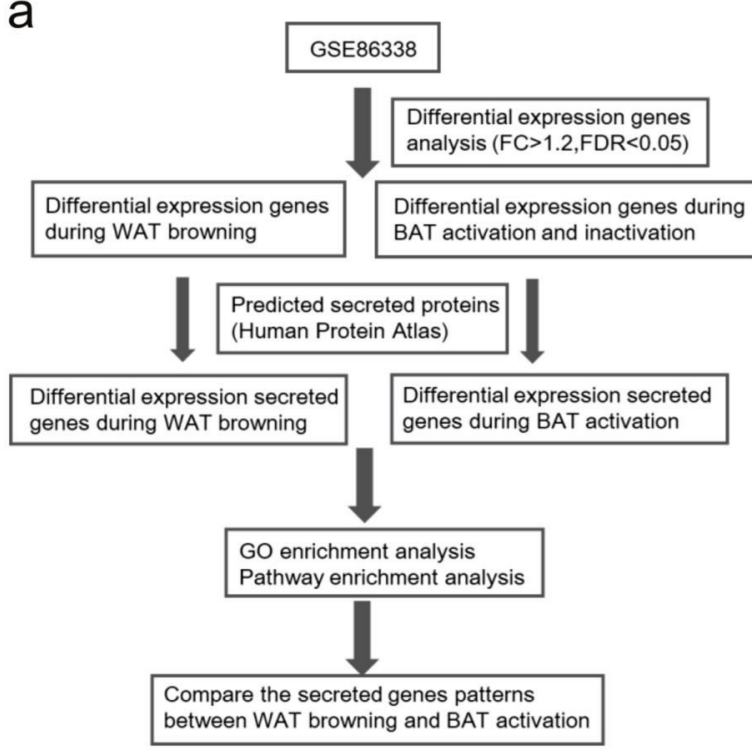

d

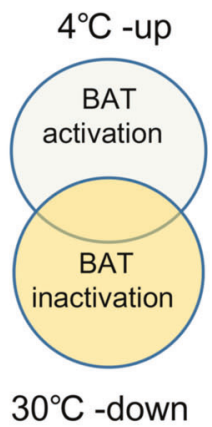

e

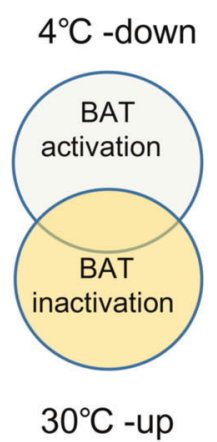

b
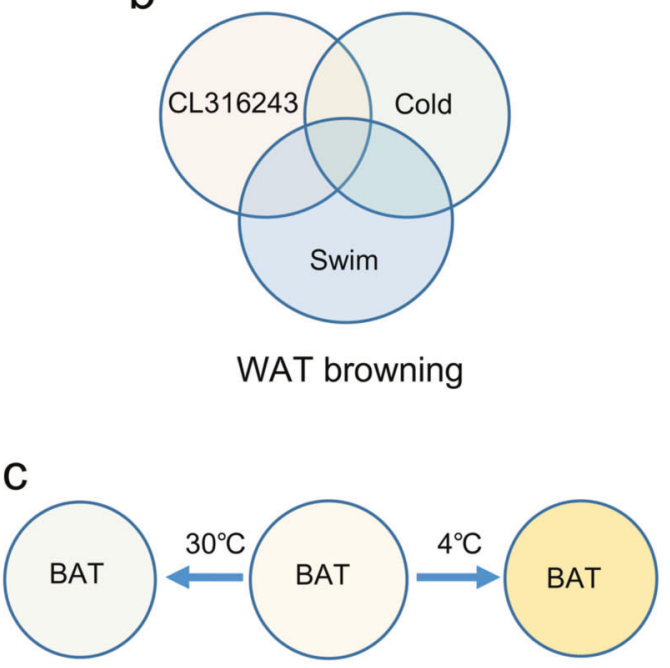

BAT inactivation BAT activation

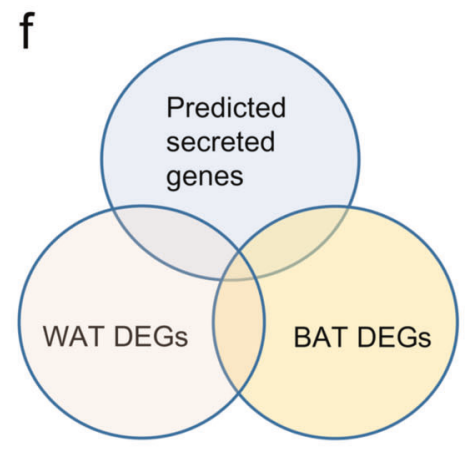

Fig. 1 Identification of DEPSGs during WAT browning and BAT activation using GEO dataset GSE86338 and predicted secreted protein lists. a Flowchart describing the process of data extraction and processing. $\mathbf{b}$ Venn analysis of DEGs during WAT browning induced by the three treatments. c Schematic representation of the two treatments $\left(6 \mathrm{~h}\right.$ of cold exposure or $30^{\circ} \mathrm{C}$ for 7 days) used to induce contrasting thermal shifts in BAT. d, e Overlap of DEGs due to contrasting thermal shifts in BAT. $\mathbf{f}$ Venn diagram comparing the overlap among DEGs of browning WAT, activated BAT, and predicted secreted genes

with DMEM medium and plated onto 24-well plates. For the induction of brown adipocytes, two days after reaching confluence, the SVF cells were incubated with medium that contained $5 \mu \mathrm{g} / \mathrm{mL}$ insulin, $0.5 \mathrm{mM}$ isobutylmethylxanthine, $1 \mu \mathrm{M}$ dexamethasone, $1 \mathrm{nM} \mathrm{T3}$, and $1 \mu \mathrm{M}$ rosiglitazone for 2 days and were subsequently cultured in medium with insulin, T3, and rosiglitazone for an additional 4 days.

Mice and treatments

Male C57BL/6 mice, 5 weeks old, were purchased from Shanghai SLAC Laboratory Animal Company and maintained with a $12 \mathrm{~h}$ light-dark cycle. One week after arrival, the mice were randomly divided into different groups according to the experiment. For the browning experiment, the mice were subjected to cold exposure $\left(4^{\circ} \mathrm{C}\right)$ for $24 \mathrm{~h}$ or 7 days, respectively. All animal procedures were approved by the Animal Care Committee of Shanghai Jiao Tong University.

Immunohistochemistry

Adipose tissue was fixed by immersion in $4 \%$ paraformaldehyde and then dehydrated, cleared, and paraffin embedded. The paraffin sections were obtained. To inactivate endogenous peroxidase, the sections were immersed in $0.3 \%$ hydrogen peroxide solution. The sections were blocked with goat serum to reduce nonspecific staining and then incubated with anti-UCP1 (1:500, ab10983) and anti-Clstn3 (1:200, ab101955) antibodies at $4{ }^{\circ} \mathrm{C}$ overnight. The HRP-conjugated secondary antibody was goat anti-rabbit IgG (PV-6001, ZSGB-BIO, Beijing, China). The sections were counterstained with hematoxylin.

Statistical analysis

All data are presented as the means \pm SEM for three independent experiments. Individual comparisons were performed using Student's $t$ test, and multiple comparisons were assessed using one-way ANOVA with Dunnett's post hoc test. A P-value $<0.05$ was considered statistically significant.

\section{RESULTS}

Identification of DEPSGs during WAT browning and BAT activation The gene expression profile of GSE86338 downloaded from the GEO database includes two types of adipose tissue samples from C57BL/6 mice: white adipose tissue with three widely used browning treatments (chronic cold exposure, $\beta$-adrenergic agonist treatment, and intense exercise) and brown adipose tissue with activation or inactivation treatment (acute cold exposure and 
a

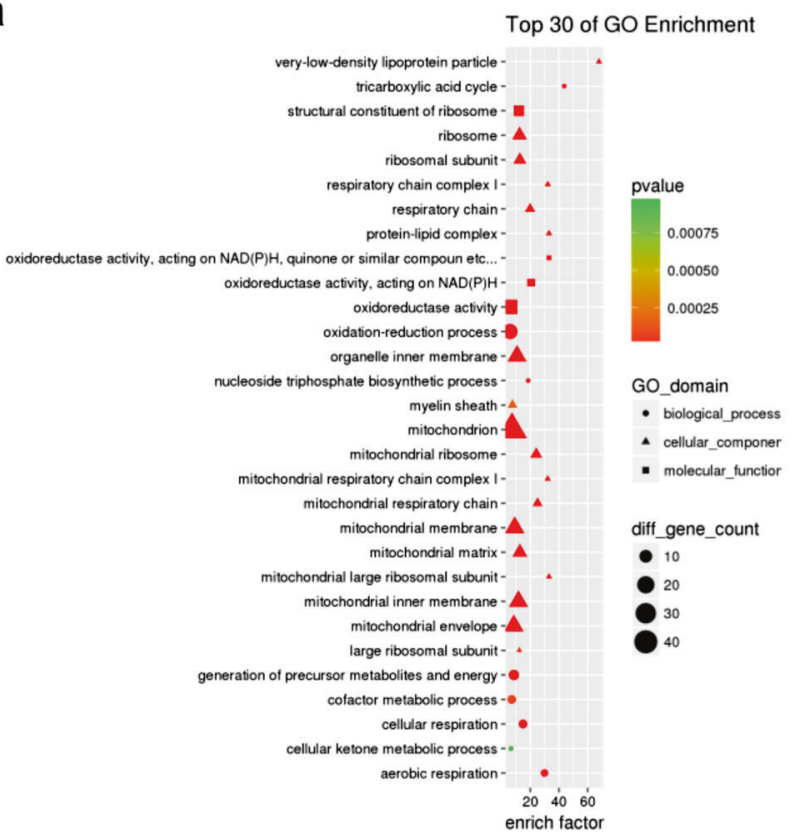

WAT browning up-regulated DEPSGs

C

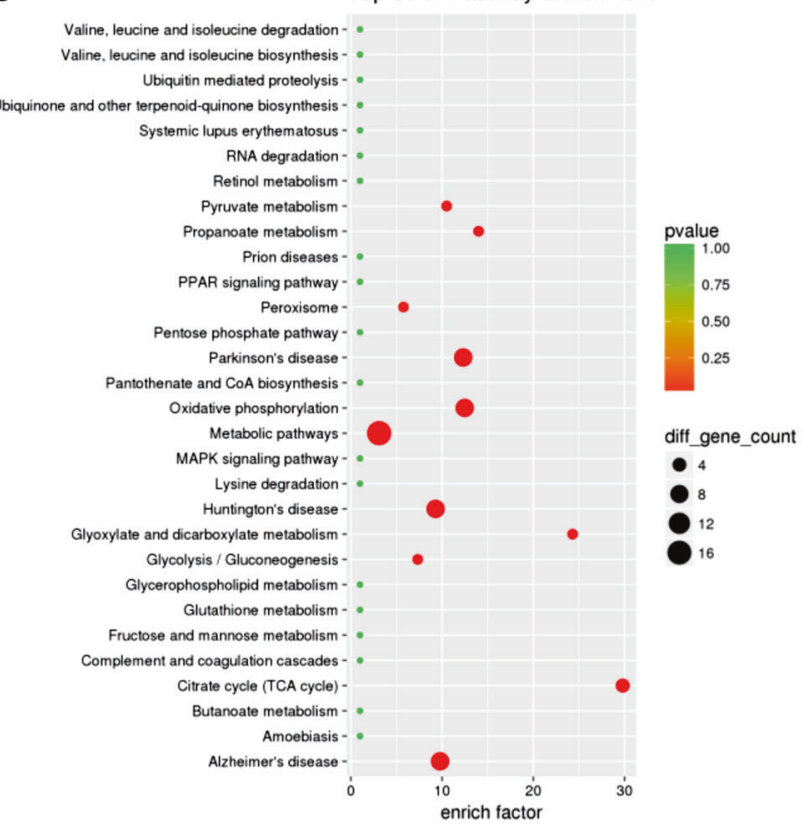

WAT browning up-regulated DEPSGs b

Top 30 of GO Enrichment

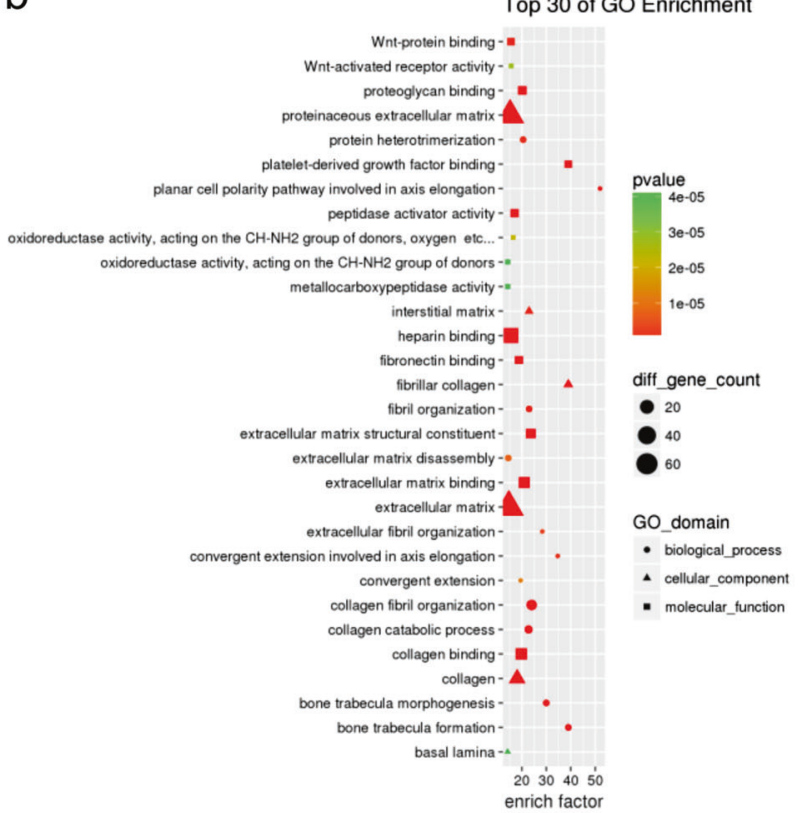

WAT browning down-regulated DEPSGs

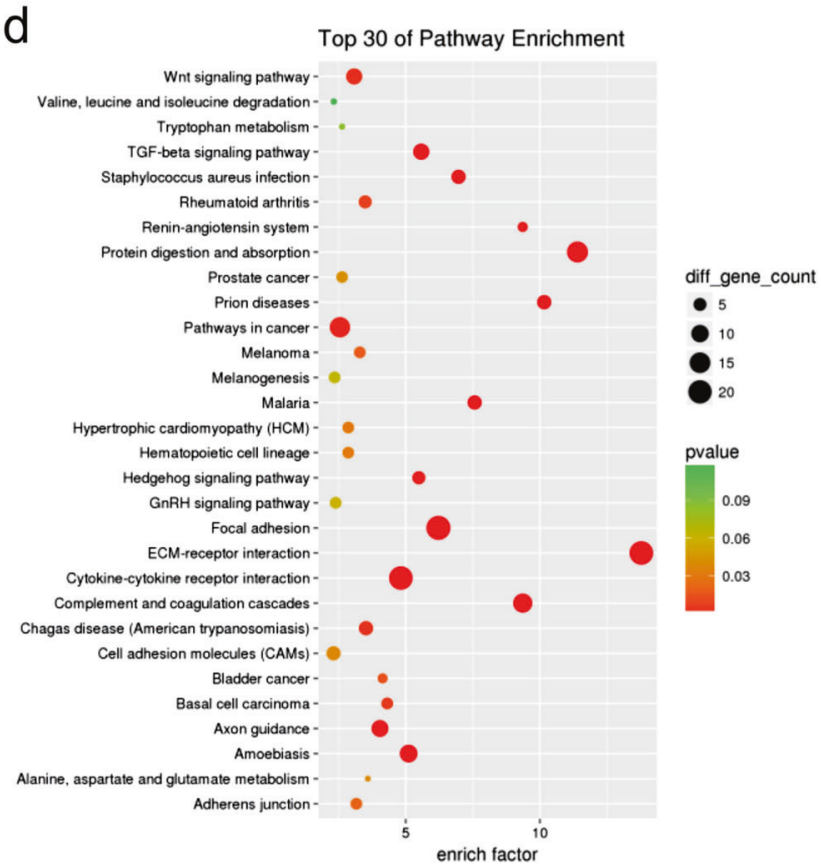

WAT browning down-regulated DEPSGs

Fig. 2 The enrichment analysis of DEPSGs of browning WAT. GO (a) and pathway (c) enrichment analyses of upregulated DEPSGs during WAT browning. GO (b) and pathway (d) enrichment analyses of downregulated DEPSGs during WAT browning

thermoneutrality, respectively) [32]. The data processing methods of GEO863338 are shown in Fig. 1a, and the detailed programming is presented in the Materials and Methods section. Comparing the DEGs during WAT browning with three conditions, we obtained an overlap of 1315 common DEGs (Fig. 1b). Eliminating 104 DEGs that were regulated in opposite directions, the remaining 1211 DEGs were used for further analysis (Supplementary Table S1).
Parallel to WAT, we examined the DEGs during BAT activation or inactivation ( $6 \mathrm{~h}$ of cold exposure or $30^{\circ} \mathrm{C}$ for 7 days). We selected the genes simultaneously regulated by these two stimuli in opposite directions (Fig. 1C-e) for further research. Overall, 1316 mRNAs downregulated in BAT activation and upregulated in BAT inactivation were identified; moreover, 1399 mRNAs upregulated in BAT activation and downregulated in BAT inactivation were identified (Supplementary Table S2). 
a

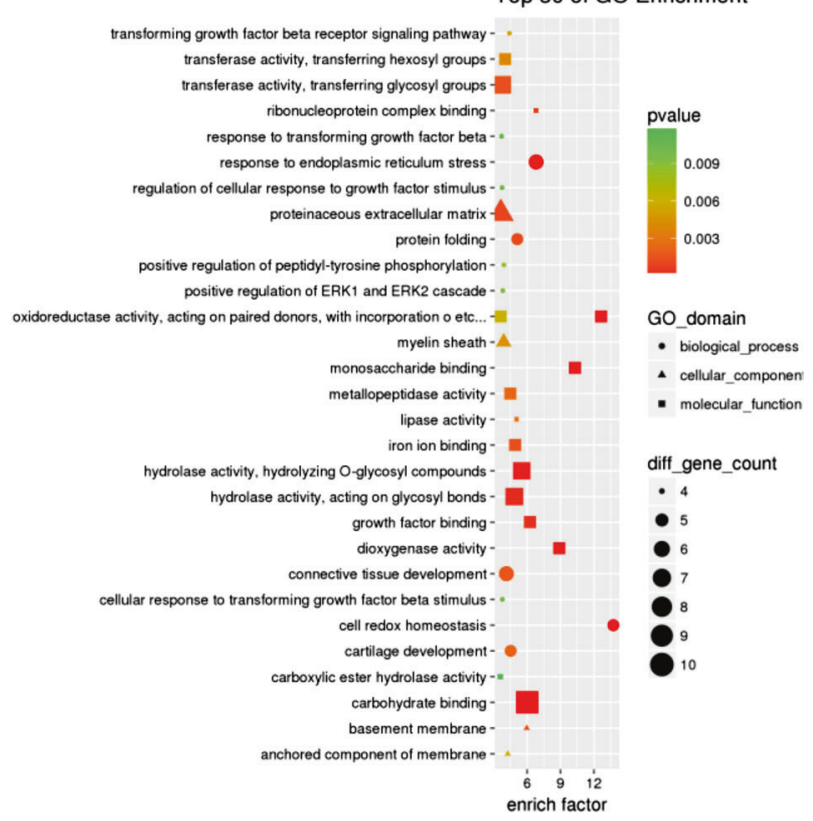

BAT activation up-regulated DEPSGs

C

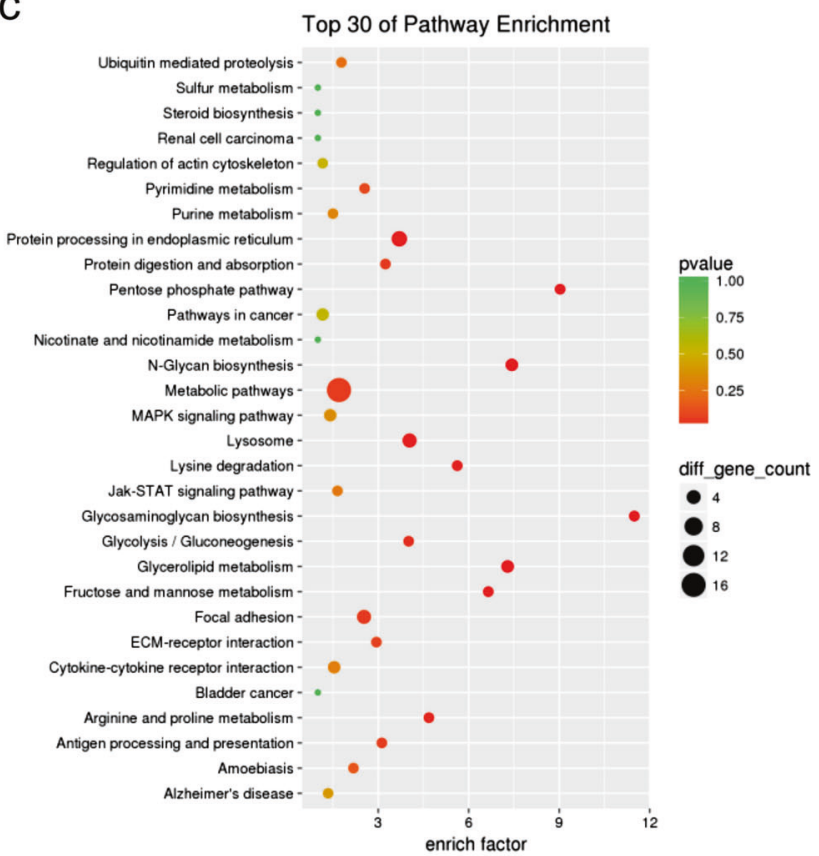

BAT activation up-regulated DEPSGs b

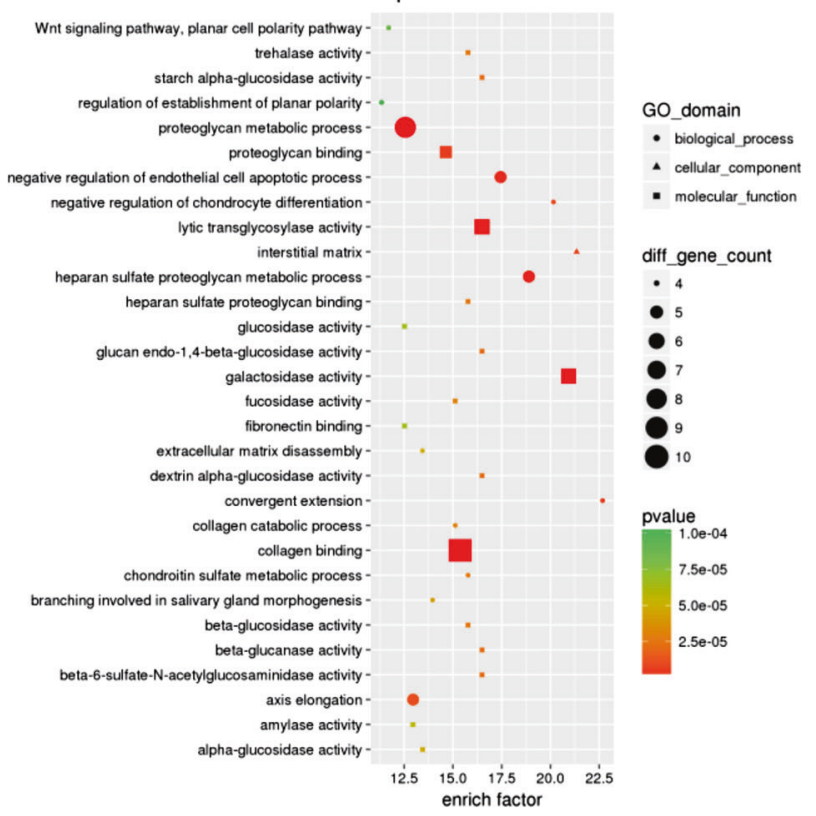

BAT activation down-regulated DEPSGs

d

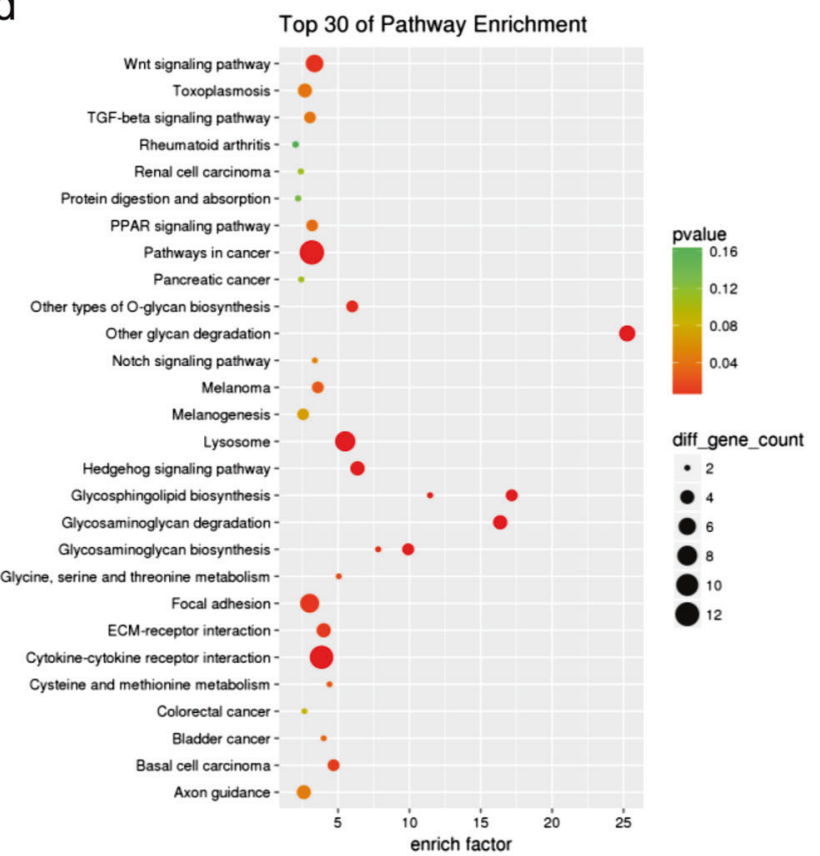

BAT activation down-regulated DEPSGs

Fig. 3 The enrichment analysis of DEPSGs of activated BAT. GO (a) and pathway (c) enrichment analyses of upregulated DEPSGs during BAT activation. GO (b) and pathway (d) enrichment analyses of downregulated DEPSGs during BAT activation

To investigate the DEPSGs, we took advantage of the predicted secreted protein list from Tissue-Based Map of Human Proteome [15] and identified 307 DEPSGs during WAT browning and 334 DEPSGs during BAT activation or inactivation, respectively (Fig. If, Supplementary Table S3). Venn analysis of the overlap between WAT and BAT showed that 65 DEPSGs were identified (Fig. 1f, Supplementary Table S3). The common DEPSGs occupied $\sim 20 \%$ of the total DEPGSs during WAT browning and BAT activation, which indicates that the secreted gene profiles of WAT browning and BAT activation had a relatively small similarity.

GO and pathway enrichment analysis of the DEPSGs during WAT browning and BAT activation

To gain further insight into the biological function of the identified DEPSGs, functional and pathway enrichment analyses were performed. We uploaded DEPSGs during WAT browning to the SBC analysis system. Gene ontology (GO) enrichment analysis 
a

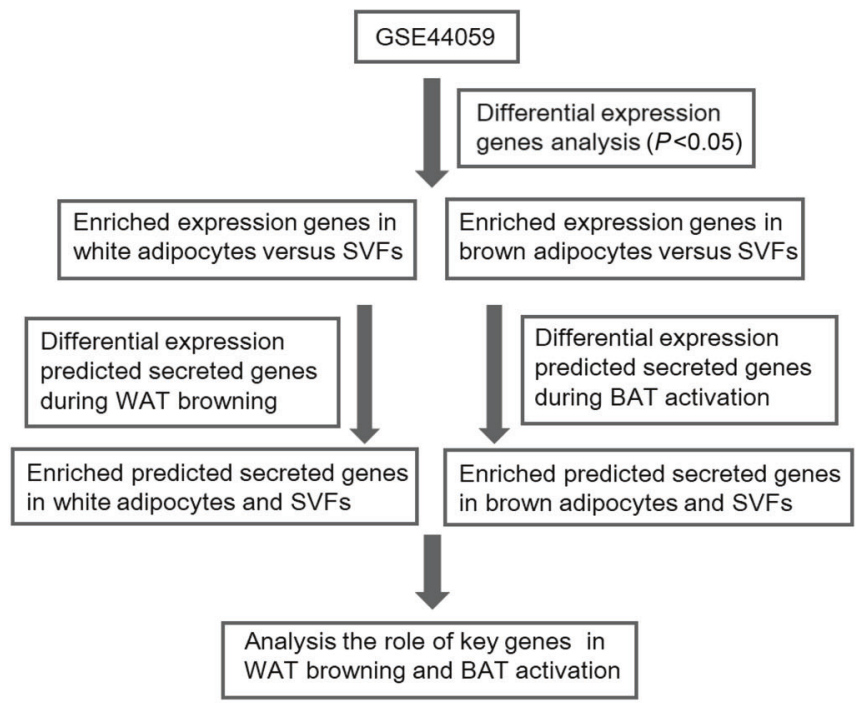

b

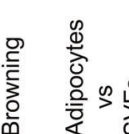

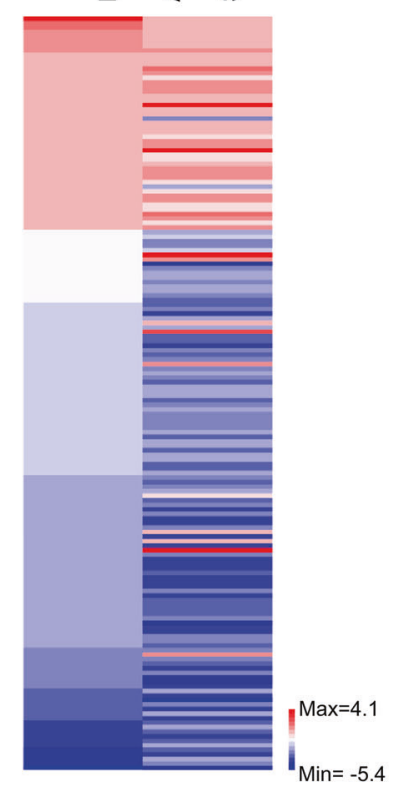

WAT

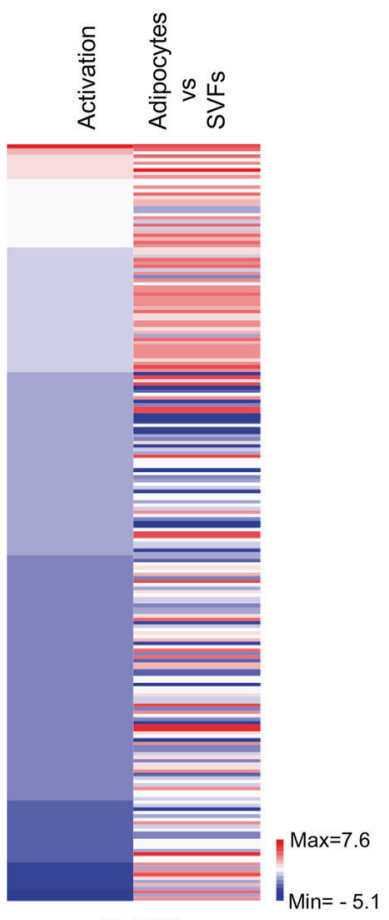

BAT

Fig. 4 Cellular distribution specificity of DEPSGs. a Flowchart of database mining strategy. Enriched DEPSGs in mature adipocytes or SVFs were identified in WAT and BAT, respectively. $\mathbf{b}$ Heat map representation of the $\log _{2}$ fold change of DEPSGs in browning WAT (left) and the relative expression levels ( $\log _{2}$ fold change ratios) of DEPSGs in WAT (right). The same type of heat map was also drawn for activated BAT

showed that the upregulated DEPSGs (62 genes, of which 62 genes were annotated with GO terms) were significantly enriched in biological themes associated with mitochondrion energy metabolism, including Mitochondrion (CC), oxidation-reduction process (BP), and oxidoreductase activity (MF) (Fig. 2a). Moreover, the downregulated DEPSGs (245 genes downregulated, of which 243 genes were annotated with GO terms) were annotated mainly by GO terms related to the extracellular domain, including extracellular matrix (CC), collagen fibril organization (BP), and collagen binding (MF) (Fig. 2b). Pathway enrichment analysis annotated upregulated DEPSGs (62 genes, of which 29 genes were annotated in the SBC analysis system) by categories related to metabolism pathways, as well as oxidative phosphorylation, while the downregulated DEPSGs (245 genes downregulated, of which 243 genes were annotated in the SBC analysis system) by categories associated with the extracellular domain (cytokine-cytokine receptor interaction and focal adhesion) (Fig. 2c, d).

We subsequently used the same methods to analyze the biological function of DEPSGs during BAT activation. The upregulated DEPSGs during BAT activation (122 genes, of which 121 genes were annotated with GO terms) were annotated by GO terms, such as carbohydrate binding (MF), proteinaceous extracellular matrix (CC) and response to ER stress (BP) (Fig. 3a). The downregulated DEPSGs (212 genes, of which 209 genes were annotated with GO terms) were enriched mainly in categories such as collagen binding (MF) and proteoglycan metabolism process (BP) (Fig. 3b). The examination of the pathway analysis indicated that the upregulated DEPSGs (122 genes, of which 53 genes were annotated in the SBA analysis system) were enriched mainly in metabolic pathways, while the downregulated DEPSGs (212 genes, 78 genes were annotated in the SBC analysis system) were annotated mainly in cytokine-cytokine receptor interaction and pathways in cancer (Fig. 3c, d). These results indicated that the enrichment analysis of the DEPSGs during WAT browning had

\begin{tabular}{lcl} 
Table 1. & Top & 10 DESGs highly expressed in white mature adipocytes \\
\hline Gene name & WAT cold exposure $\left(\log _{2} \mathrm{FC}\right)$ & Adipocytes vs SVFs $\left(\log _{2} \mathrm{FC}\right)$ \\
\hline Vnn1 & -1.81004 & 4.03 \\
Clstn3 & 0.54405 & 3.86 \\
Apoc1 & 0.702009 & 3.76 \\
Tshr & -0.75075 & 3.61 \\
St6galnac5 & -1.06722 & 2.67 \\
Slc2a5 & 0.866748 & 2.33 \\
Fam132a & 0.389696 & 2.17 \\
Apcdd1 & -2.37274 & 1.9 \\
Sdhb & 0.931638 & 1.85 \\
Msra & 0.384735 & 1.81
\end{tabular}

a neat segregated biological pattern. Thus, the upregulated biological themes were mainly associated with energy metabolism, while the downregulated biological themes were mainly associated with the extracellular matrix. The enrichment analysis of the DEPSGs during BAT activation did not show such an obvious segregated biological pattern similar to WAT browning, further highlighting that the secreted gene profiles of WAT browning and BAT activation were somewhat different.

From the GO and pathway enrichment analysis, we found that most of the DEPSGs were annotated with extracellular matrix (ECM). The ECM is an obligatory component of the adipose tissue stroma that provides structural support and biochemical signals to maintain proper adipose tissue function. Extracellular matrix remodeling may play a beneficial role in adipose tissue thermogenesis. In addition, our results showed that inflammation and the fibrosis-related signaling pathways (TGF $\beta$ and Wnt signaling pathways) were decreased during WAT browning and BAT 
Table 2. Top 10 DESGs highly expressed in brown mature adipocytes

\begin{tabular}{lcl} 
Gene name & BAT cold exposure $\left(\log _{2} \mathrm{FC}\right)$ & Adipocytes vs SVFs $\left(\log _{2} \mathrm{FC}\right)$ \\
\hline Clstn3 & 1.849704 & 3.44 \\
II17re & -2.22518 & 2.82 \\
Ltbp2 & -1.26776 & 2.41 \\
Glb112 & -1.27115 & 2.4 \\
Adipoq & -0.40483 & 2.29 \\
Dio2 & 7.58599 & 2.14 \\
Vegfb & -0.50319 & 2.14 \\
Echdc1 & 0.296433 & 2.11 \\
Cdnf & -2.9189 & 2.08 \\
Calr3 & -0.64257 & 2.07 \\
\hline
\end{tabular}

activation, thus indicating that the process of adipose tissue browning shifts the extracellular microenvironment into a good and healthy state.

Cellular distribution of the upregulated and downregulated DEPSGs

As a distinctive biological pattern of enrichment analysis was observed for the upregulated and downregulated DEPSGs during WAT browning, we sought to determine the cellular distribution of the two types of influenced genes. We took advantage of the GSE44059 datasets that contain microarray gene expression data performed in mature adipocytes and SVFs of the interscapular brown, inguinal subcutaneous and visceral epididymal adipose tissue depots of young adult male C57BL/6 to explore the specific cellular enrichment of the two groups of genes (upregulated and downregulated genes) in isolated WAT or BAT cellular fractions [25]. GEO2R (https://www.ncbi.nlm.nih.gov/geo/geo2r/) was a

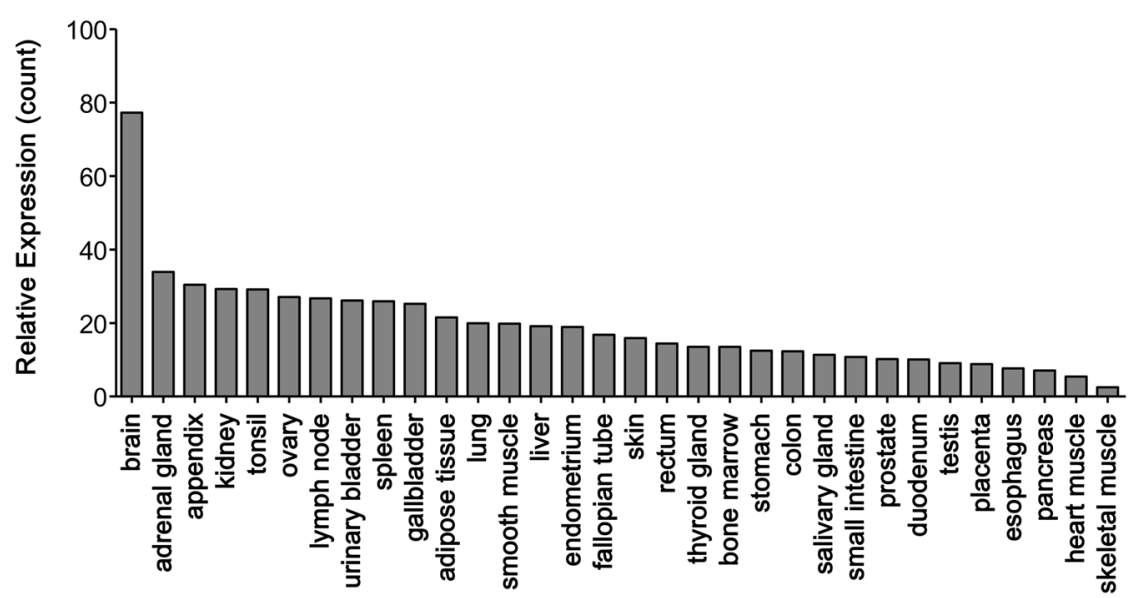

b

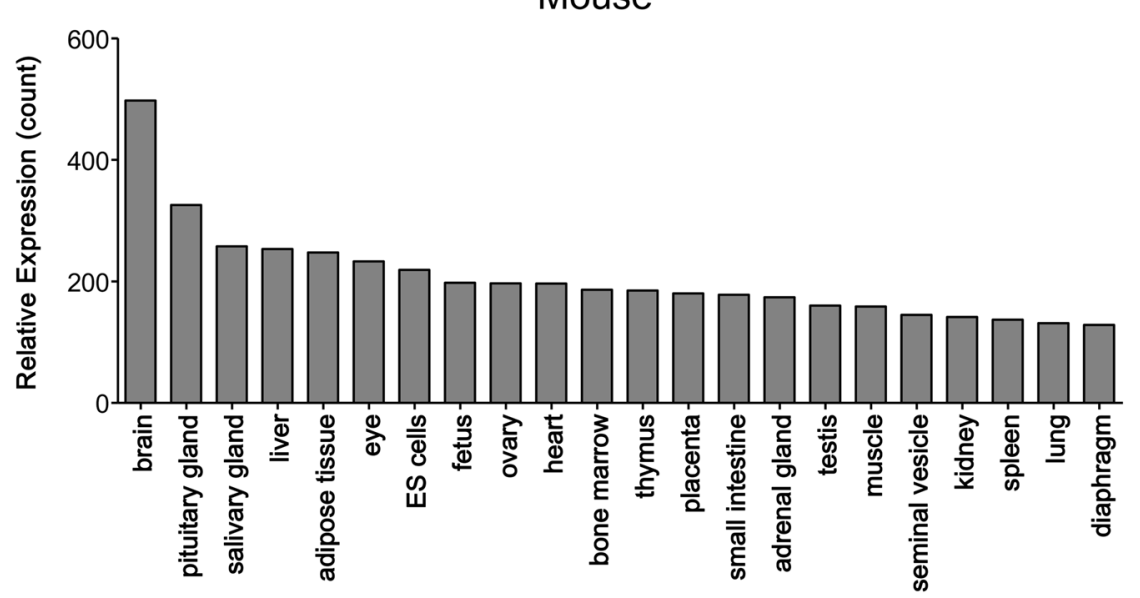

C
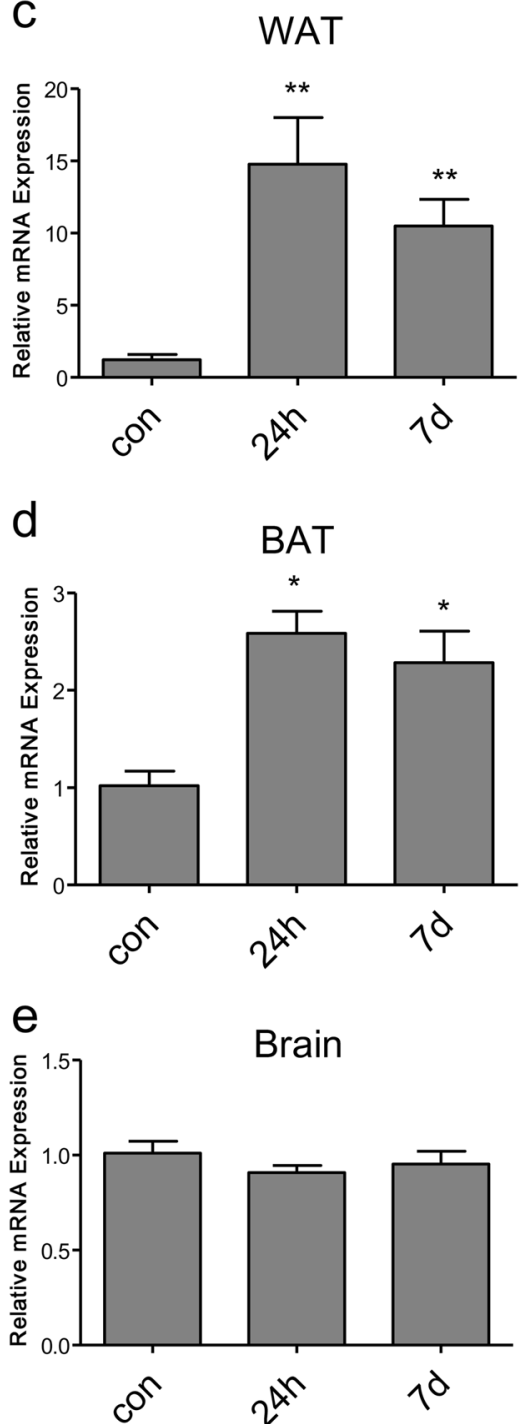

Fig. 5 Tissue expression profile of Clstn3 and the validation of its expression during WAT browning and BAT activation. $\mathbf{a}$, $\mathbf{b}$ Tissue expression profile of Clstn3 gene in human (a) and mouse (b) tissues. Human data were obtained from The Human Protein Atlas (http://www.proteinatlas. org). Mouse data were obtained from the GEO dataset GSE9954. c-e Q-PCR analysis of clstn3 expression in WAT (c), BAT (d), and brain (e) after $24 \mathrm{~h}$ or 7 days cold exposure. The results are presented as the mean \pm SEM. Statistical comparisons were made using one-way ANOVA, and multiple comparisons were conducted using Dunnett's post hoc test. ${ }^{*} P<0.05$ and ${ }^{* *} P<0.01$ versus control group 
a

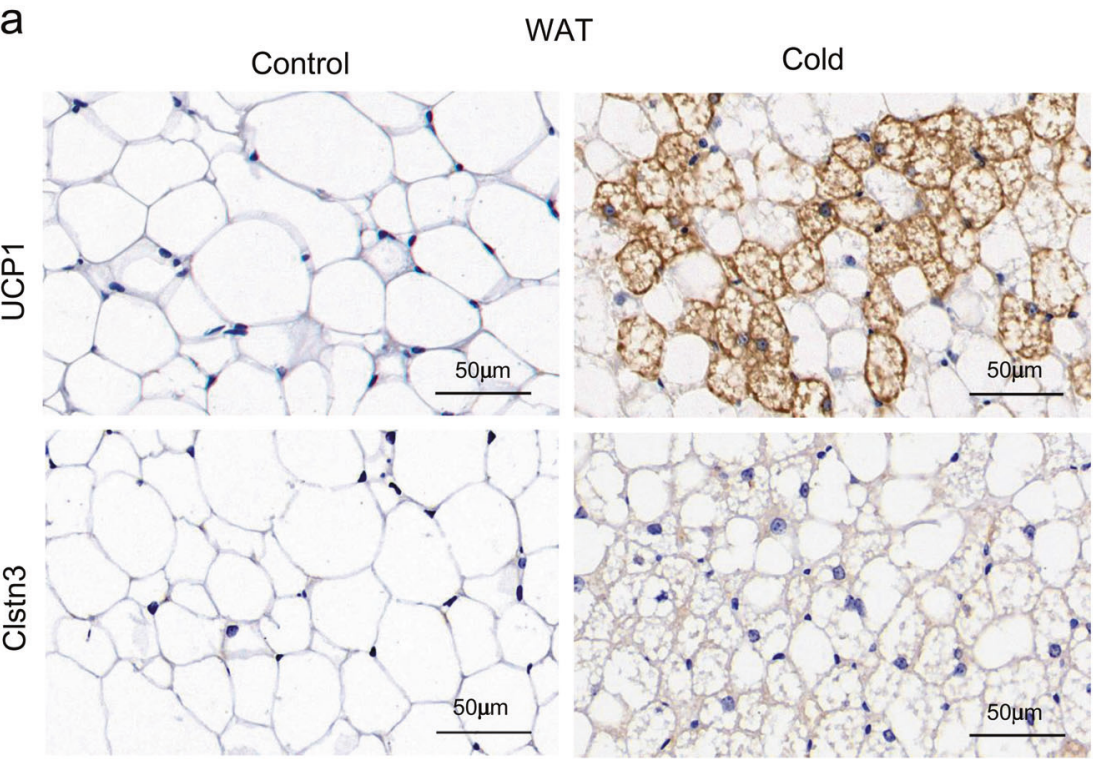

b

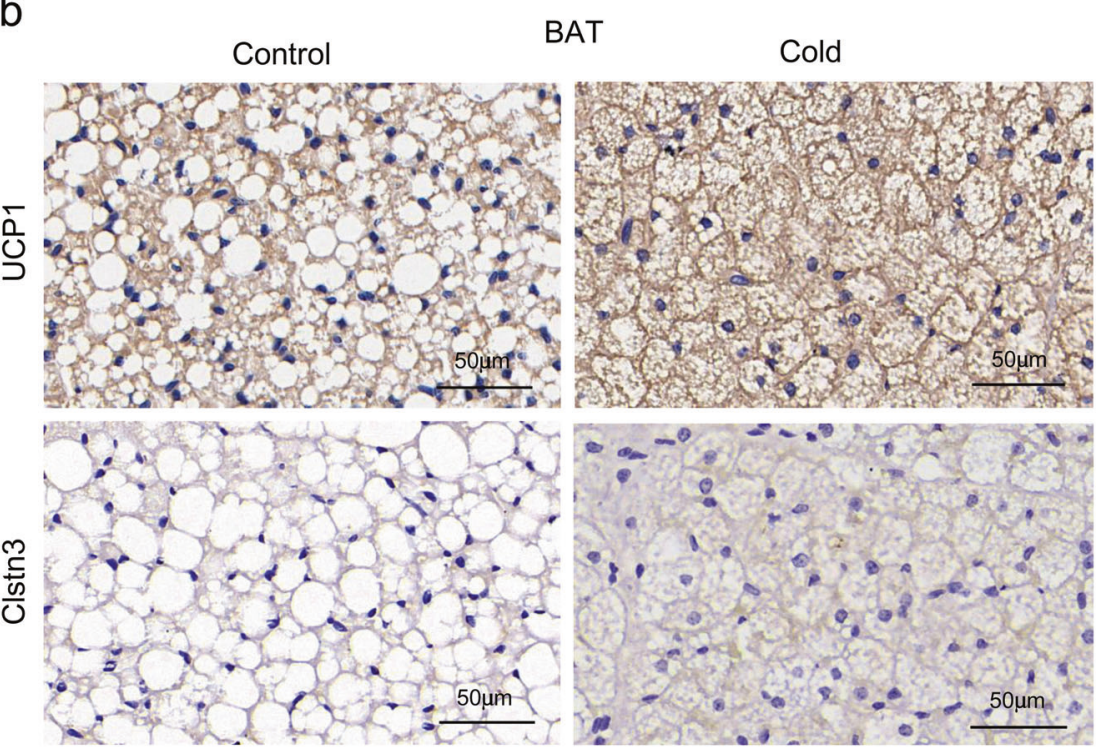

Fig. 6 Detection of Clstn3 protein by immunohistochemistry in WAT and BAT. Immunohistochemistry of inguinal WAT (a) and BAT (b) without or with cold exposure for 7 days using UCP1 and Clstn3 antibody. Images are representative of two to three different mice per group

applied to screen DEGs enriched in mature adipocytes versus SVFs [26]. A $P$-values $<0.05$ was set as the cut-off criterion. $\log _{2} \mathrm{FC}>0$ indicated that the genes were mainly expressed in mature adipocytes, while $\log _{2} \mathrm{FC}<0$ indicated that the genes were mainly expressed in SVFs. The detailed program of data processing is shown in Fig. 4a.

After eliminating genes that did not appear in the microarray data GSE44059 and genes filtered by the cut-off criterion ( $P$-value $<0.05$ ), we obtained an overlap of 166 genes between WAT browning DEPSGs and cellular distribution specifically DEGs (Supplementary Table S4). Comparing the gene expression changes $\left(\log _{2} \mathrm{FC}\right)$ during WAT browning and the cellular distribution of specific genes, we found that upregulated DEPSGs annotated with biological themes related to mitochondrion energy metabolism were predominantly expressed in mature adipocytes, while downregulated DEPSGs annotated with biological themes associated with the extracellular domain were mainly expressed in the SVF of WAT (Fig. 4b). We also used the same methods to analyze the DEPSGs of BAT activation (Supplementary
Table S5). However, for BAT activation, the DEPSGs did not show an obvious manifestation similar to WAT browning (Fig. 4b).

Clstn3 may function as a key molecule in neuro-adipose synapse formation or remodeling during WAT browning and BAT activation

We then sought to identify the key molecules that can reflect the cross-talk between mature adipocytes and other types of cells. As mature adipocytes are the most important functional cells in WAT and BAT and the composition of fat cells is relatively single compared to SVFs, we choose mature adipocytes for further analysis. We listed the top 10 genes that were highly expressed in white mature adipocytes (Table 1) and brown mature adipocytes (Table 2) and found that Clstn3 was the only gene highly expressed in both white and brown fat cells. We explored the Human Protein Atlas database (www.proteinatlas.org) and GSE9954 to view the tissue expression profiles of human and mouse Clstn3 [15]. As shown in Fig. 5a, b, the Clstn3 gene was predominantly expressed in the brain and widely expressed in 


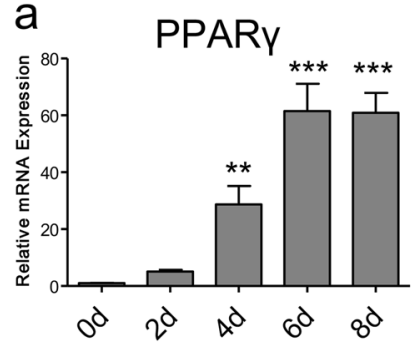

Induced white adipocytes

e

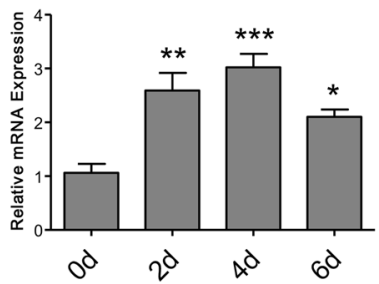

Induced beige adipocytes b

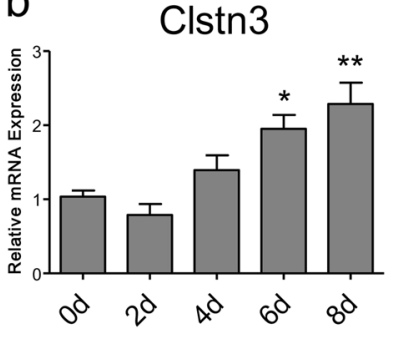

Induced white adipocytes
C

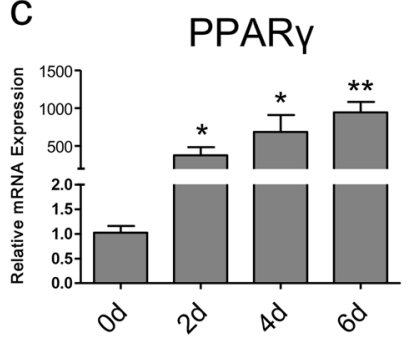

Induced beige adipocytes d

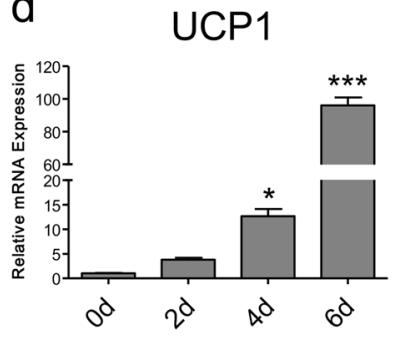

Induced beige adipocytes f

UCP1

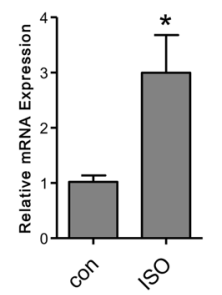

White adipocytes g Clstn3

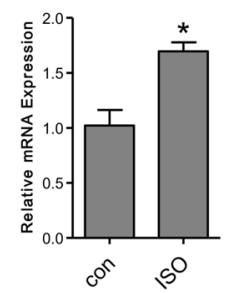

White adipocytes $\mathrm{h}$

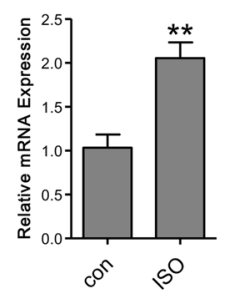

Beige adipocytes i

\section{Clstn3}

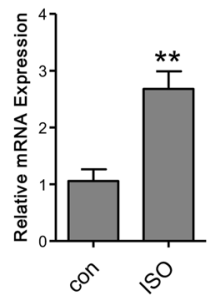

Beige adipocytes

Fig. 7 The expression of Clstn3 during adipocyte differentiation and after exposure to ISO in vitro. a-e Q-PCR analyses of Clstn3, PPARy, and UCP1 expression during differentiation of 3T3-L1 to white adipocytes (a, b) and SVFs to brown adipocytes (c-e). $\mathbf{f}-\mathbf{i}$ Relative mRNA levels of Clstn3 and UCP1 in white adipocytes (f, $\mathbf{g})$ differentiated from 3T3-L1 preadipocytes with or without ISO treatment (1 $\mu \mathrm{m}$ for $6 \mathrm{~h})$ and in brown adipocytes $(\mathbf{h}, \mathbf{i})$ differentiated from SVFs with or without ISO treatment $(10 \mu \mathrm{m}$ for $6 \mathrm{~h})$. The results are presented as the mean \pm SEM. Statistical comparisons of induced white and beige adipocytes were made using one-way ANOVA, and multiple comparisons were performed using Dunnett's post hoc test. ${ }^{*} P<0.05,{ }^{*} P<0.01$, and ${ }^{* *} P<0.001$ versus 0 days group. Statistical comparisons between two groups were made using the unpaired Student's $t$ test. ${ }^{*} P<0.05,{ }^{* *} P<0.01$, and ${ }^{* * *} P<0.001$ versus control group

other tissues and organs with relatively low levels. The RNA-Seq data and Q-PCR results showed that the Clstn3 expression was increased during WAT browning and BAT activation, whereas the expression levels of Clstn3 in the brain did not change when mice were exposed to cold (Fig. 5c, d).

To further explore the expression changes of Clstn3 protein during browning and identify its localization in adipose tissue, we performed immunohistochemistry experiments. As shown in Fig. 6a, the staining for Clstn3 and UCP1 are significantly increased in WAT after cold exposure. Cold-induced mature adipocytes with multilocular lipid droplets generally exhibit cytoplasm and membrane staining. The expression of Clstn3 in BAT is higher than in WAT. Similar to WAT, the expressions of Clstn3 and UCP1 are increased in BAT after cold exposure for 7 days (Fig. 6b).

Clstn 3 was identified as a synaptogenic adhesion molecule, and the increased expression of Clstn3 may reflect new synaptic structure formation during WAT browning and BAT activation [21, 22]. To evaluate the function of Clstn3 in mature adipocytes, the 3T3-L1 preadipocytes were differentiated toward mature white adipocytes and SVF cells were differentiated toward beige adipocytes using the protocols described in the Materials and Methods section. We found that the Clstn3 gene expression was increased and in parallel with the induction of PPARY and UCP1 when preadipocytes were differentiated to mature adipocytes [33, 34] (Fig. 7a-e). To simulate the effects of cold exposure on mouse fat cells, white and beige adipocytes were stimulated with isoproterenol (ISO) in vitro. Here, we found that ISO treatment could induce white and beige adipocyte Clstn3 expression in parallel with the induction of UCP1 [34-36] (Fig. 7f-i). Recent studies have reported that Clstn3 promotes synapse development $[21,22]$. Thus, we speculated that Clstn3 may promote neuroadipose synapse formation or remodeling during WAT browning and BAT activation. We also analyzed other candidate genes (Supplementary Table S6) that have been reported to induce presynaptic differentiation in heterologous synapse-formation assays [21]. We found that Clstn3 was the only gene for which the expression changed during WAT browning and BAT activation, which indicates its important roles in this process.

\section{DISCUSSION}

The enhancement of BAT activity and the promotion of WAT browning, which are believed to increase an organism's energy expenditure, are attractive therapeutic strategies for obesity and its complications. Defining the signals and the function changes of the extracellular microenvironment can advance our understanding of the browning process. Targeting sympathetic innervation in WAT and BAT represents an attractive concept to combat obesity and its complications. Neuro-adipose synaptic junctions are the key microstructures that mediate sympathetic innervation in adipose tissue. Our study identified DEPSGs during WAT browning and BAT activation, which can represent the extracellular microenvironment remodeling. Using bioinformatic methods, we found that the secreted gene expression profiles between WAT browning and BAT activation had a relatively small similarity. In addition, enrichment analysis showed that upregulated DEPSGs during WAT browning were mainly related to mitochondrion energy metabolism, while downregulated DEPSGs were generally associated with the extracellular domain. Furthermore, the cellular distribution analysis demonstrated that upregulated secreted genes were predominantly expressed in mature adipocytes, while downregulated secreted genes were mainly expressed in the SVFs of WAT. Through this data analysis, combined with a preliminary experimental exploration, we successfully identified that Clstn3 may act as a key molecule in neuro-adipose synapse formation or remodeling during WAT browning and BAT activation.

Synapse formation and elimination remain active throughout the lifetime of an organism [37]. To date, the research on synapses 
mainly focuses on nerve tissue, while research on adipose tissue is relatively rare. The neuro-adipose synaptic microstructure is very important to mediate sympathetic inputs to adipose tissues. However, the developmental signals and molecular mechanisms that guide nerve terminals to mature adipocytes remain incompletely understood. Here, our study showed that the expression of Clstn3, a synaptogenic CAM, increases during cold exposure, which implies the formation and remodeling of synapses in adipose tissue. We suggest this is a manifestation of the adaptive function of adipose tissue to cope with changes in the external environment. In addition, previous studies have explored the function of Clstn3, which is believed to promote synapse development [21, 22]. Combined with our experimental results, these findings indicate that Clstn3 may play a key role in the formation and development of synapses in adipose tissue in response to a cold challenge.

The thermogenic function of WAT browning and BAT activation has been recognized by many researchers. However, the extracellular microenvironment changes during WAT browning and BAT activation have received less attention. Here, our study primarily focuses on the changes of the extracellular microenvironment. Previous research has investigated the extracellular microenvironment mainly via mass spectrometry to detect cell supernatant or tissue supernatant. This method cannot detect the cross-talk between cells. Our study employed a different strategy of using predicted secreted protein profiles, which can largely reflect the extracellular microenvironment remodeling. This method is relatively convenient, and it is capable of detecting the connection changes between cells. As the prediction principle of the secreted protein is based on signal peptides, some of these predicted genes inevitably reside inside the endoplasmic reticulum, Golgi or endosomes. Overall, it does not affect our subsequent analysis and outcome judgment. Our research results suggested that Clstn3 plays an important role in the formation and remodeling of synapses in adipose tissue. However, further animal experiments are essential to verify this conclusion. In addition, the specific mechanism by which Clstn3 plays a role in adipose tissue remains unclear, and further research is required.

The current study has indicated the DEPSG profiles during WAT browning and BAT activation. Key GO terms and pathways were identified using enrichment analysis. The cellular distribution specificity analysis showed that upregulated and downregulated secreted genes have segregated cell distribution patterns. Most importantly, our work provided new evidence at the molecular levels to support the increased nerve innervation in adipose tissue and suggests that the synapse-organizing molecule CLSTN3 may play a key role in neuro-adipose synapse formation or remodeling during WAT browning and BAT activation. Our study may provide new drug targets for the treatment of obesity and other metabolic disorders.

\section{ACKNOWLEDGEMENTS}

This work was supported by grants from the National Natural Science Foundation of China (No. 81471085, No. 81670778, and No. 81603476) and the Innovation Fund for PhD Students from Shanghai Jiao Tong University School of Medicine (BXJ201841).

\section{ADDITIONAL INFORMATION}

The online version of this article (https://doi.org/10.1038/s41401-019-0211-2) contains supplementary material, which is available to authorized users.

Competing interests: The authors declare no competing interests.

\section{REFERENCES}

1. Unamuno X, Gomez-Ambrosi J. Adipokine dysregulation and adipose tissue inflammation in human obesity. Eur J Clin Invest. 2018;48(9):e12997.
2. Expert panel on the identification, evaluation, and treatment of overweight in adults. Clinical guidelines on the identification, evaluation, and treatment of overweight and obesity in adults: executive summary. Am J Clin Nutr. 1998;68:899-917.

3. Frontini A, Cinti S. Distribution and development of brown adipocytes in the murine and human adipose organ. Cell Metab. 2010;11:253-6.

4. Harms $M$, Seale P. Brown and beige fat: development, function and therapeutic potential. Nat Med. 2013;19:1252-63.

5. Jespersen NZ, Larsen TJ, Peijs L, Daugaard S, Homoe P, Loft A, et al. A classical brown adipose tissue mRNA signature partly overlaps with brite in the supraclavicular region of adult humans. Cell Metab. 2013;17:798-805.

6. Xue B, Coulter A, Rim JS, Koza RA, Kozak LP. Transcriptional synergy and the regulation of Ucp1 during brown adipocyte induction in white fat depots. Mol Cell Biol. 2005;25:8311-22.

7. Bartelt A, Heeren J. Adipose tissue browning and metabolic health. Nat Rev Endocrinol. 2014;10:24-36.

8. Cannon B, Nedergaard J. Brown adipose tissue: function and physiological significance. Physiol Rev. 2004;84:277-359.

9. Frayn KN, Karpe F, Fielding BA, Macdonald IA, Coppack SW. Integrative physiology of human adipose tissue. Int J Obes Relat Metab Disord. 2003;27:875-88.

10. Pope BD, Warren CR, Parker KK, Cowan CA. Microenvironmental control of adipocyte fate and function. Trends Cell Biol. 2016;26:745-55.

11. Jiang $H$, Ding $X$, Cao $Y$, Wang $H$, Zeng W. Dense intra-adipose sympathetic arborizations are essential for cold-induced beiging of mouse white adipose tissue. Cell Metab. 2017;26:686-92.e3.

12. Vitali A, Murano I, Zingaretti MC, Frontini A, Ricquier D, Cinti S. The adipose organ of obesity-prone C57BL/6J mice is composed of mixed white and brown adipocytes. J Lipid Res. 2012;53:619-29.

13. Muzik O, Mangner TJ, Leonard WR, Kumar A, Granneman JG. Sympathetic innervation of cold-activated brown and white fat in lean young adults. J Nucl Med. 2017;58:799-806.

14. Zeng W, Pirzgalska RM, Pereira MM, Kubasova N, Barateiro A, Seixas E, et al. Sympathetic neuro-adipose connections mediate leptin-driven lipolysis. Cell. 2015;163:84-94.

15. Uhlen M, Fagerberg L, Hallstrom BM, Lindskog C, Oksvold P, Mardinoglu A, et al. Proteomics. Tissue-based map of the human proteome. Science. 2015;347:1260419.

16. Petersen TN, Brunak S, von Heijne G, Nielsen H. SignalP 4.0: discriminating signal peptides from transmembrane regions. Nat Methods. 2011;8:785-6.

17. Kall L, Krogh A, Sonnhammer EL. Advantages of combined transmembrane topology and signal peptide prediction--the Phobius web server. Nucleic Acids Res. 2007;35:W429-32.

18. Viklund H, Bernsel A, Skwark M, Elofsson A. SPOCTOPUS: a combined predictor of signal peptides and membrane protein topology. Bioinformatics. 2008;24:2928-9.

19. Blobel G, Dobberstein B. Transfer of proteins across membranes. I. Presence of proteolytically processed and unprocessed nascent immunoglobulin light chains on membrane-bound ribosomes of murine myeloma. J Cell Biol. 1975;67:835-51.

20. Walter P, Ibrahimi I, Blobel G. Translocation of proteins across the endoplasmic reticulum. I. Signal recognition protein (SRP) binds to in-vitro-assembled polysomes synthesizing secretory protein. J Cell Biol. 1981;91:545-50.

21. Um JW, Pramanik G, Ko JS, Song MY, Lee D, Kim H, et al. Calsyntenins function as synaptogenic adhesion molecules in concert with neurexins. Cell Rep. 2014;6:1096-109.

22. Pettem KL, Yokomaku D, Luo L, Linhoff MW, Prasad T, Connor SA, et al. The specific alpha-neurexin interactor calsyntenin-3 promotes excitatory and inhibitory synapse development. Neuron. 2013;80:113-28.

23. Lu Z, Wang Y, Chen F, Tong H, Reddy MV, Luo L, et al. Calsyntenin-3 molecular architecture and interaction with neurexin 1alpha. J Biol Chem. 2014;289:34530-42.

24. Clough E, Barrett T. The gene expression omnibus database. Methods Mol Biol. 2016;1418:93-110.

25. Rosenwald M, Perdikari A, Rulicke T, Wolfrum C. Bi-directional interconversion of brite and white adipocytes. Nat Cell Biol. 2013;15:659-67.

26. Barrett T, Wilhite SE, Ledoux P, Evangelista C, Kim IF, Tomashevsky M, et al. NCBI GEO: archive for functional genomics data sets-update. Nucleic Acids Res. 2013:41:D991-5

27. Burgess DJ. Gene expression: spatial characterization of proteomes. Nat Rev Genet. 2015;16:129.

28. Thul PJ, Lindskog C. The human protein atlas: a spatial map of the human proteome. Protein Sci. 2018;27:233-44.

29. The Gene Ontology Consortium. The gene ontology (GO) project in 2006. Nucleic Acids Res. 2006;34:D322-6.

30. Ashburner M, Ball CA, Blake JA, Botstein D, Butler H, Cherry JM, et al. Gene ontology: tool for the unification of biology. The Gene Ontology Consortium. Nat Genet. 2000;25:25-9.

31. Ogata H, Goto S, Sato K, Fujibuchi W, Bono H, Kanehisa M. KEGG: Kyoto encyclopedia of genes and genomes. Nucleic Acids Res. 1999;27:29-34. 
32. Bai Z, Chai XR, Yoon MJ, Kim HJ, Lo KA, Zhang ZC, et al. Dynamic transcriptome changes during adipose tissue energy expenditure reveal critical roles for long noncoding RNA regulators. PLoS Biol. 2017;15:e2002176.

33. Cristancho AG, Lazar MA. Forming functional fat: a growing understanding of adipocyte differentiation. Nat Rev Mol Cell Biol. 2011;12:722-34.

34. Chu DT, Malinowska E, Gawronska-Kozak B, Kozak LP. Expression of adipocyte biomarkers in a primary cell culture models reflects preweaning adipobiology. J Biol Chem. 2014;289:18478-88.
35. Collins S, Cao W, Robidoux J. Learning new tricks from old dogs: beta-adrenergic receptors teach new lessons on firing up adipose tissue metabolism. Mol Endocrinol. 2004;18:2123-31.

36. Mowers J, Uhm M, Reilly SM, Simon J, Leto D, Chiang SH, et al. Inflammation produces catecholamine resistance in obesity via activation of PDE3B by the protein kinases IKKe and TBK1. eLife. 2013;2:e01119.

37. Missler M, Sudhof TC, Biederer T. Synaptic cell adhesion. Cold Spring Harb Perspect Biol. 2012;4:a005694. 